\title{
MiR-23a Functions as a Tumor Suppressor in Osteosarcoma
}

\author{
Yu He Chunqing Meng Zengwu Shao Hong Wang Shuhua Yang \\ Department of Orthopaedics, Union Hospital, Tongji Medical College, Huazhong University \\ of Science and Technology, Wuhan, P.R. China
}

\section{Key Words}

Osteosarcoma $\cdot \mathrm{miR}-23 \mathrm{a} \cdot$ Methylation $\bullet \mathrm{RUNX2} \cdot \mathrm{CXCL12}$

\begin{abstract}
Background: Osteosarcoma is the most common primary bone malignancy in children and adolescents, and the pathogenesis of this cancer remains unclear. Therefore, the discovery of new biomarkers for the diagnosis, prognosis, and treatment of osteosarcoma remains an important but unmet clinical need. Method: Quantitative real-time PCR was carried out to examine the expression of miR-23a. Methylation-specific PCR was performed to evaluate the DNA methylation status of the miR-23a promoter. Cell proliferation, migration, and invasion were examined by cell counting assays, wound healing assays, and cell invasion assays, respectively. Western blot analysis and luciferase reporter assays were performed to identify miR-23 target genes. Nude mice were used to investigate the function of miR-23a in vivo. Results: The expression of miR-23a was decreased in osteosarcoma cells and tissues compared to normal controls. The promoter region of the miR-23a gene was hypermethylated in osteosarcoma cells, and demethylase treatment increased the expression of miR-23a. The ectopic expression of miR-23a led to retarded proliferation, migration, and invasion of osteosarcoma cells, whereas the depletion of miR-23a resulted in the opposite effects. MiR-23a suppressed the transcription of RUNX2 and CXCL12 by binding to the $3^{\prime}$ UTRs of these mRNAs. The cellular function of miR-23a is RUNX2/CXCL12-dependent, and the overexpression of RUNX2 or CXCL12 rescued the impaired cell growth, migration, and invasion induced by miR-23a. Nude mouse experiments indicated that miR-23a may inhibit the proliferation of osteosarcoma cells in vivo. Conclusion: We identified miR-23a as a tumor suppressor in osteosarcoma. Our data clarify the mechanism of osteosarcoma progression and demonstrated the potential for exploiting miR-23a as a diagnostic marker for osteosarcoma.
\end{abstract}

Copyright @ 2014 S. Karger AG, Basel

Y. He and C. Meng contributed equally to this work. 


\section{Introduction}

Osteosarcoma (OS) is the most prevalent primary bone malignancy [1]. Approximately $53 \%$ of OS occurs in children and adolescents (ages 0 - 24), that is more common in males than in females [2,3]. Despite its rarity, OS is the third leading cause of cancer-related death in the paediatric age group behind lymphomas and brain tumors $[4,5]$. The aetiology of OS is unclear, and the molecular mechanisms of OS progression are poorly-understood [6]. Therefore, the discovery of new biomarkers for the diagnosis, prognosis, and treatment of OS remains an important but unmet clinical need.

MicroRNAs (miRNAs) are small non-coding RNA molecules (18 - $25 \mathrm{nt}$ ) that are involved in the regulation of gene expression at the transcriptional or post-transcriptional levels. MiRNAs are incorporated into the RISC complex and base paired to the 3'-untranslated region (3' UTR) of target genes to exert their regulatory effects [7]. MiRNAs regulate several types of physiological and pathological processes, including embryogenesis, cell maintenance, lineage determination, and normal cell physiology [8-11]. Studies have also shown that miRNAs are involved in tumorigenesis and cancer progression. Many miRNAs are deregulated in various cancer subtypes, such as breast cancer, colon cancer, gastric cancer, hepatocellular cancer, lung cancer, and OS [12-20]. It has been reported that miRNAs function both as oncogenes and tumor suppressor genes, depending upon the roles of their mRNA targets. Therefore, miRNA have been classified as oncogenic, tumor-suppressive, or context-dependent miRNAs [21].

The regulation of miRNA expression occurs at several levels, including the transcriptional, post-transcriptional, and epigenetic levels [22, 23]. Epigenetic regulation is an important aspect of gene regulation [24]. The promoter hypermethylation of classic tumor suppressor genes is commonly observed in cancers, whereas the hypermethylation of oncogenic promoters is frequently occurs in non-transformed cells [25]. DNA methylation occurs predominantly at $\mathrm{CpG}$ dinucleotides, and acts as a relatively stable gene silencing mechanism [26]. The key enzymes that participate in DNA methylation are a family of DNA methyltransferases (DNMTs) that catalyze the transfer of methyl groups to the 5' position of cytosine bases in the CpG dinucleotide [27].

In our previous study, we identified altered miRNA expression profiles in OS using a microarray-based approach. In the present study, we analysed the expression of miR-23a in OS cells and clinical tissues. We found that downregulation of miR-23a in OS cells and tissues was due to hypermethylation of the miR-23a promoter region. Introduction of miR-23a mimics inhibited the growth of OS cells, whereas miR-23a inhibitors resulted in accelerated cell proliferation. Similarly, miR-23a mimics inhibited the migration and invasion of OS cells while miR-23a inhibitors exhibited the opposite effect. We observed that miR-23a regulated the mRNA and protein expression of RUNX2 and CXCL12 by directly binding to the 3' UTR of these genes. Finally, we validated our in vitro results using nude mouse models, demonstrating that miR-23a suppressed tumor growth in vivo. Our results demonstrated the possibility of utilizing miR-23a as a useful diagnostic, prognostic, and therapeutic biomarker for OS.

\section{Materials and Methods}

\section{Cell lines}

Saos-2, MG-63, HOS MNNG/HOS Cl \#5, U-2 OS osteosarcoma cell lines, and murine pre-osteoblast MC3T3-E1 cells were purchased from the ATCC (Manassas, VA, USA). Murine bone marrow stroma cells (BMSCs) and murine pre-chondrocyte ATDC5 cells were purchased from CHI Scientific, Inc (Jiangsu, China). All cells were cultured in RPMI 1640 medium containing $10 \%$ fetal bovine serum (FBS) at $37^{\circ} \mathrm{C}, 5 \% \mathrm{CO}_{2}$ with saturated humidity.

Tissue specimens

The 20 paired of osteosarcoma specimens and adjacent tissues used in this study were obtained from surgically excised samples from the Department of Orthopaedics, Union Hospital. After pathological 
verification, an osteosarcoma sample and an adjacent tissue sample were obtained from each patient. This study was carried out with the approval of the Ethics Committee of Tongji Medical College, Huazhong University of Science and Technology.

\section{DNA and RNA extraction}

DNA was extracted from the osteosarcoma cell lines and clinical samples using a DNA extraction kit (Promega, USA) according to the manufacturer's instructions. RNA was extracted from the tissue and cell samples using Trizol (Invitrogen, USA).

\section{Quantitative real-time PCR analysis}

Total RNA was isolated from the cell lines and clinical samples using TRIzol (Invitrogen Life Technologies, USA). After quantification, $2 \mu \mathrm{g}$ of total RNA was reverse-transcribed with the BulgeLoop $^{\mathrm{TM}}$ hsa-miR-23a qRT-PCR Primer Set (Guangzhou RiboBio, China) according to the manufacturer's instructions. The efficiency of the primers was examined to ensure optimal sample amplification. Real-time PCR was performed using a SYBR Green PCR Master mix in an ABI PRISM 7900HT Sequence Detection System (Applied Biosystems, USA). For the analysis of miR-23a target genes, the primers used were as follows: 5' TTACAGACCCCAGGCAGGCACA 3' and 5'TCCATCAGCGTCAACACCATCA3' for RUNX2; 5' TGCTGCCTCTACCTGACACT 3' and 5' GTCCTGATACAGAGCTGGCA 3' for CXCL12.5' AAGGTCGGAGTCAACGGATT 3' and 5' CTGGAAGATGGTGATGGGATT 3' for GAPDH. The constitutively expressed housekeeping gene, GAPDH, was used as an endogenous control to correct for any potential variations in RNA loading or reaction efficiency.

\section{Methylation Assay}

Genomic DNA was isolated from osteosarcoma cell lines and clinical samples using a DNA extraction kit (Promega, USA) according to the manufacturer's instructions. Two micrograms of genomic DNA was treated with bisulphate, and polymerase chain reaction (PCR) was performed using external nested PCR primers corresponding to a region that is unaffected by the methylation status. Equal aliquots of the amplicon were then amplified with internal nested PCR primers corresponding to either a methylated promoter region or a region that is unaffected by the methylation status. The PCR products were electrophoresed on a $1.2 \%$ agarose gel, and the PCR product of the internal 2 was used as loading control.

\section{Cell proliferation assay}

Cells were transfected with a miR-23a agomir, antagomir, or the corresponding controls (Guangzhou RiboBio, China) at $40 \%$ confluence according to the manufacturer's instructions. At the indicated time points, cells were collected for counting cell numbers using Countess (Invitrogen, USA).

\section{Wound healing assay}

Cells were transfected with miR-23a mimic, inhibitor, or the corresponding controls (Guangzhou RiboBio, China) according to the manufacturer's instructions, and cultured until monolayers were formed. The monolayers were scratched with a $200-\mu$ l pipette tip and washed to remove the detached cells. The cells were maintained in serum-free media for 24 hours, and the wounded areas were photographed. The cell migration distances were measured to indicate the migration ability of differently treated cells.

\section{Cell invasion assay}

Cells were treated as previous described, and $1 \times 10^{5}$ cells were resuspended in $300 \mu \mathrm{l}$ of serum-free media and added onto a filter coated with basement membrane Matrigel. Bio-Coat cell migration chambers (BD Biosciences, USA) were supplemented with RPMI 1640 with 20\% FBS. After $24 \mathrm{~h}$ of incubation, the cells on the upper surface of the filter were removed, and the invading cells on the lower surface were fixed with $5 \%$ glutaric dialdehyde and Giemsa stained to quantify the number of cells. The number of invading cells in 5 randomly chosen microscopic fields was counted to determine the invasive ability of differently treated cells.

\section{Western blot analysis}

Cells were treated as previous described, and $24 \mathrm{~h}$ later cells were lysed using lysis buffer containing $20 \mathrm{mM}$ Tris (pH 7.4), $150 \mathrm{mM} \mathrm{NaCl}, 1 \%$ TritonX-100, 0.5\% (w/v) sodium deoxycholate, $0.1 \%$ SDS, $5 \mathrm{mM}$ EDTA, $10 \mu \mathrm{g} / \mathrm{ml}$ leupeptin, $10 \mu \mathrm{g} / \mathrm{ml}$ aprotinin, and $1 \mathrm{mM}$ phenylmethylsulphonyl fluoride. The protein 
concentration of the cell lysates was measured by a bicinchoninic acid (BCA) protein assay (Pierce, IL, USA), and the proteins were separated by SDS-PAGE and transferred to Hybond ECL nitrocellulose membranes (Amersham Biosciences, NJ, USA). The antibodies used for immunoblotting were as follows: anti-RUNX2 rabbit monoclonal antibody, anti-CXCL12 rabbit monoclonal antibody, and anti-GAPDH rabbit monoclonal antibody, all of these antibodies were purchased from Cell Signaling Technology (USA).

\section{Reporter Assay}

Cells were cotransfected with a wild-type or mutant of luciferase reporter containing the promoter region of the RUNX2 or CXCL12 genes, and either miR-23a mimic or inhibitor. Cell lysates were harvested $24 \mathrm{~h}$ later and luciferase activity was measured using the Dual-Luciferase Reporter System (Promega, USA) according to the manufacturer's instructions. The luciferase activities were normalized to that of Renilla luciferase. The results are expressed as the means \pm SDs of at least three independent experiments.

\section{Animal experiments}

The care and use of experimental animals were approved by the ethical committee of Tongji Medical College, Huazhong University of Science and Technology, and adhered to institutional guidelines and ethical standards. Thirty-two 5-week-old male nude mice (BALB/cA) were obtained from the Shanghai Institute of Materia Medica, Chinese Academy of Sciences. MG-63 or Saos-2 cells were grown to log phase and inoculated into nude mice subcutaneously at the right lateral breech. The nude mice were randomly divided into the following four groups: MG-63 with agomir control, MG-63 with agomir, Saos-2 with antagomir control, and Saos-2 with antagomir. After 5 days of inoculation, the agomir or antagomir was injected intratumorally at $60 \mathrm{mg} / \mathrm{Kg}$ every two weeks. Tumor latency and growth were observed daily after tumor cell injection. Tumor volume was calculated using the following formula: tumor volume $=$ long radius $\times$ short radius ${ }^{2} \times 0.5$.

\section{Statistical analysis}

Unless otherwise indicated, all data were analyzed using SPSS 11.0 and presented as the mean \pm SEM. A typical image from at least three similar experiments was administered. Statistical analysis was determined using $t$-tests. A value of $\mathrm{P}<0.05$ was considered to be statistically significant.

\section{Results}

\section{MiR-23a is downregulated in OS cells and tissues}

In our previous study, we used Agilent human miRNA microarrays to screen out several differentially expressed miRNAs between OS and normal tissues. To further validate our results, we measured the expression of miRNAs in OS cells and tissues using quantitative real-time RT-PCR. The cells lines utilized in this section included Saos-2, MG-63, HOS MNNG/ $\mathrm{HOS} \mathrm{Cl} \mathrm{\# 5,} \mathrm{and} \mathrm{U-2} \mathrm{OS} \mathrm{osteosarcoma} \mathrm{cells.} \mathrm{As} \mathrm{shown} \mathrm{in} \mathrm{Fig.} 1 \mathrm{~A}$, the expression levels of miR23a were significant lower in OS cells ( $\mathrm{p}<0.05, \mathrm{p}<0.01$ in MG-63 and U-2 OS cells) than in the BMSCs, MC3T3-E1, and ADTC5 cells. We next analyzed the expression level of miR-23a in 20 pairs of OS samples and adjacent tissues. After the pathological confirmation of the disease, paired of OS samples and normal adjacent tissues were obtained from each patient. As shown in Fig. 1 B, we observed that miR-23a expression was lower in all tumors, whereas all normal controls exhibited at least 2.5 -fold higher levels of miR-23a $(\mathrm{p}<0.05)$. These findings indicated that the downregulation of miR-23a is a frequent event in human OS samples.

The deregulation of miR-23a is due to the methylation of the miR-23a promoter

To reveal the mechanisms that underlies the deregulation of miR-23a in OS cells and tissues, we analyzed the promoter region of the miR-23a gene. After finding that the promoter region of miR-23a is rich in CpG islands, we examined whether CpG methylation accounts for the low expression of miR-23a in OS. The OS cells indicated previously were harvested for methylation analysis. As shown in Fig. 2 A, the methylation status of the miR-23a promoter was positive in OS cells, especially in MG-63 and U-2 OS cells, and the methylation status of the miR-23a promoter was consistent with the low expression of miR-23a in these cell 
A

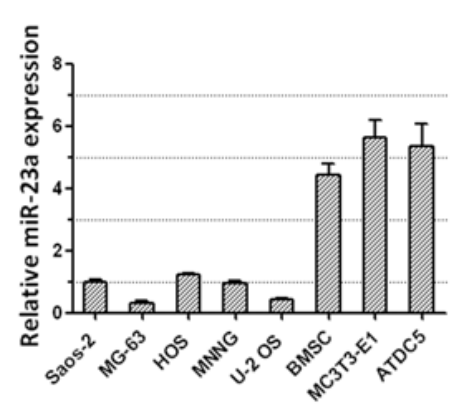

B

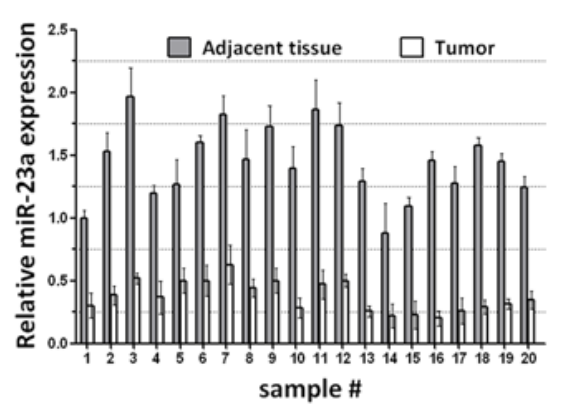

Fig. 1. The expression levels of miR-23a in osteosarcoma cell lines and tissues. (A) The expression of miR23a in osteosarcoma cell lines. The Saos-2, MG-63, HOS MNNG/HOS Cl \#5, and U-2 OS osteosarcoma cell lines and MC3T3-E1, BMSCs, and ATDC5 cells were cultured as previously described. The cells were collected for quantitative real-time PCR analysis. (B) The expression of miR-23a in osteosarcoma tissues. Twenty pairs of osteosarcoma and adjacent tissues were collected for quantitative real-time PCR analysis with the approval of the Ethics Committee of Tongji Medical College, Huazhong University of Science and Technology.

Fig. 2. The downregulation of miR-23a is due to the the methylation of the miR23a promoter. (A) The methylation of the miR-23a promoter in osteosarcoma cell lines. The indicated os cells were collected for methylation analysis as described in the Materials \& Methods section. (B) The methylation of the miR-23a promoter in osteosarcoma tissues. (C) The efficiency of 5-ZAZ treatment. The indicated cells were treated with 5-ZAZ for 7 days and the methylation status of the miR-23a promoter was determined. (D) The expression of miR-23a was significantly increased after 5-ZAZ treatment. The indicated cells were treated with 5-ZAZ for 7 days and the cells were collected for quantitative real-time PCR analysis. *, $\mathrm{p}<0.05$ vs the untreated groups.

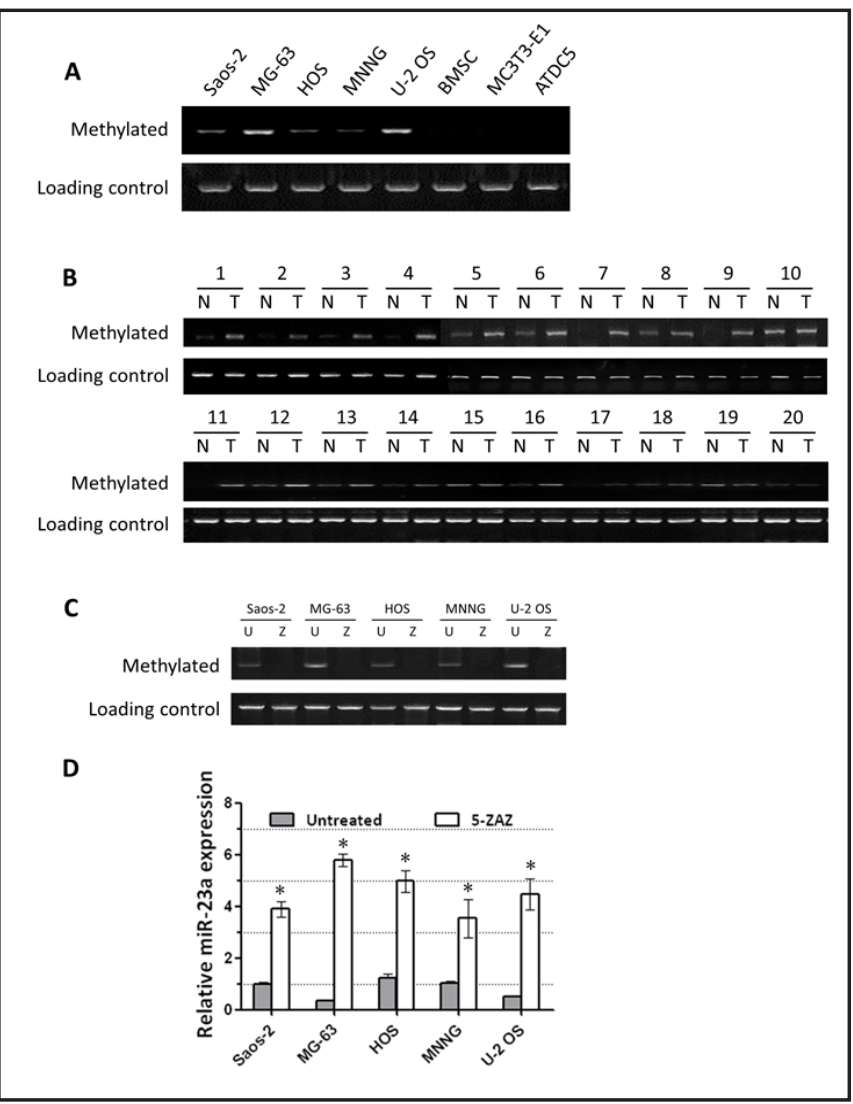

lines. The methylation status of the miR-23a promoter was also analysed in the OS samples and adjacent tissues. As shown in Fig. 2 B, methylation was observed in the adjacent normal controls, but the signals were much lower than those of the corresponding tumor tissues. A DNA demethylase, 5-ZAZ, was used to treat OS cells to reverse the methylation status of the miR-23a promoter. As expected, the methylation of the miR-23a promoter nearly disappeared after 5-ZAZ treatment (Fig. $2 \mathrm{C}$ ). The expression of miR-23a was strikingly elevated after 5-ZAZ treatment (Fig. $2 \mathrm{D}, \mathrm{p}<0.05$ ), suggesting that the hypermethylation of miR-23a leads to the reduced expression of miR-23a in OS cells. 
Fig. 3. MiR-23a inhibited OS cell proliferation, migration, and invasion. (A) Agomir of miR-23a inhibited the proliferation of MG-63 cells. MG63 cells were transfected with miR-23a agomir or a control, and the cell number was counted for 5 days. (B) Antagomir of miR-23a stimulated the proliferation of Saos- 2 cells. (C) Mimic of miR-23a inhibited the migration of MG-63 cells. MG-63 cells were transfected with miR-23a mimic or control, and the migration ability of the cells was determined by wound healing assays after 24 h. (D) Inhibitor of miR23a promoted the migration of Saos-2 cells. (E) Quantification of cell migration abilities. (F) Mimic of miR-23a inhibited the invasion of MG-63 cells, whereas inhibitor of miR-23a stimulated the invasion of Saos- 2 cells. The cells were transfected with miR-23a mimic, inhibitor or control, the cell invasion ability of the cells was determined by transwell assays after 24 h. (G) Quantification of cell invasion abilities. ${ }^{*}, \mathrm{p}<0.05$ vs the control groups.

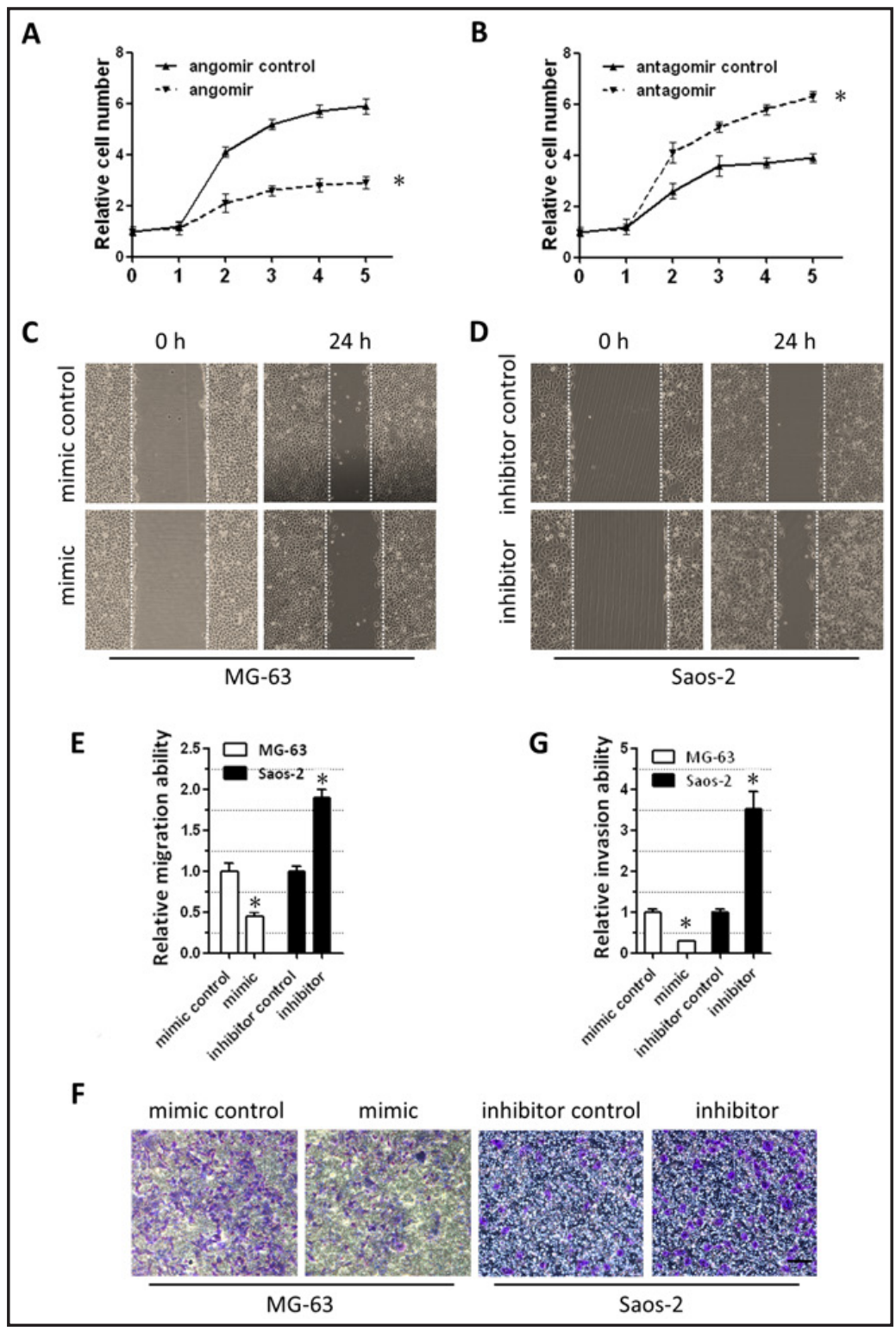

MiR-23a suppresse cell proliferation, migration, and invasion in OS cells

To test the function of miR-23a, we used the mimic (agomir) and inhibitor (antagomir) of miR-23a to perform gain-of-function and loss-of-function assays. As shown in Fig. $3 \mathrm{~A}$, after the introduction of miR-23a agomir, the proliferation of MG-63 cells was significantly increased $(\mathrm{p}<0.05)$. On the contrary, the introduction of miR-23a antagomir resulted in the stimulation of proliferation in Saos-2 cells (as indicated in Fig. 3 B, p<0.05). These data suggested that miR-23a negatively regulated cell proliferation. We next performed wound healing assays to examine the effects of the expression level of miR-23a on the migration ability of OS cells. As demonstrated in Fig. 3 C, mimic of miR-23a inhibited the migration of MG63 cells, whereas miR-23a inhibitor caused an increase in the cell migration of Saos-2 (Fig. 3 D). The distance of cell migration was calculated to determine the cell migration ability, and a quantification of cell migration ability is shown in Fig. $3 \mathrm{E}(\mathrm{p}<0.05)$. We then determined the effect of miR-23a on cell invasion using transwell assays. Consistent with the results of the migration assays, after the introduction of miR-23 mimic dramatically suppressed the invasion of MG-63 cells while miR-23a inhibitor caused a stimulation of cell invasion in Saos- 


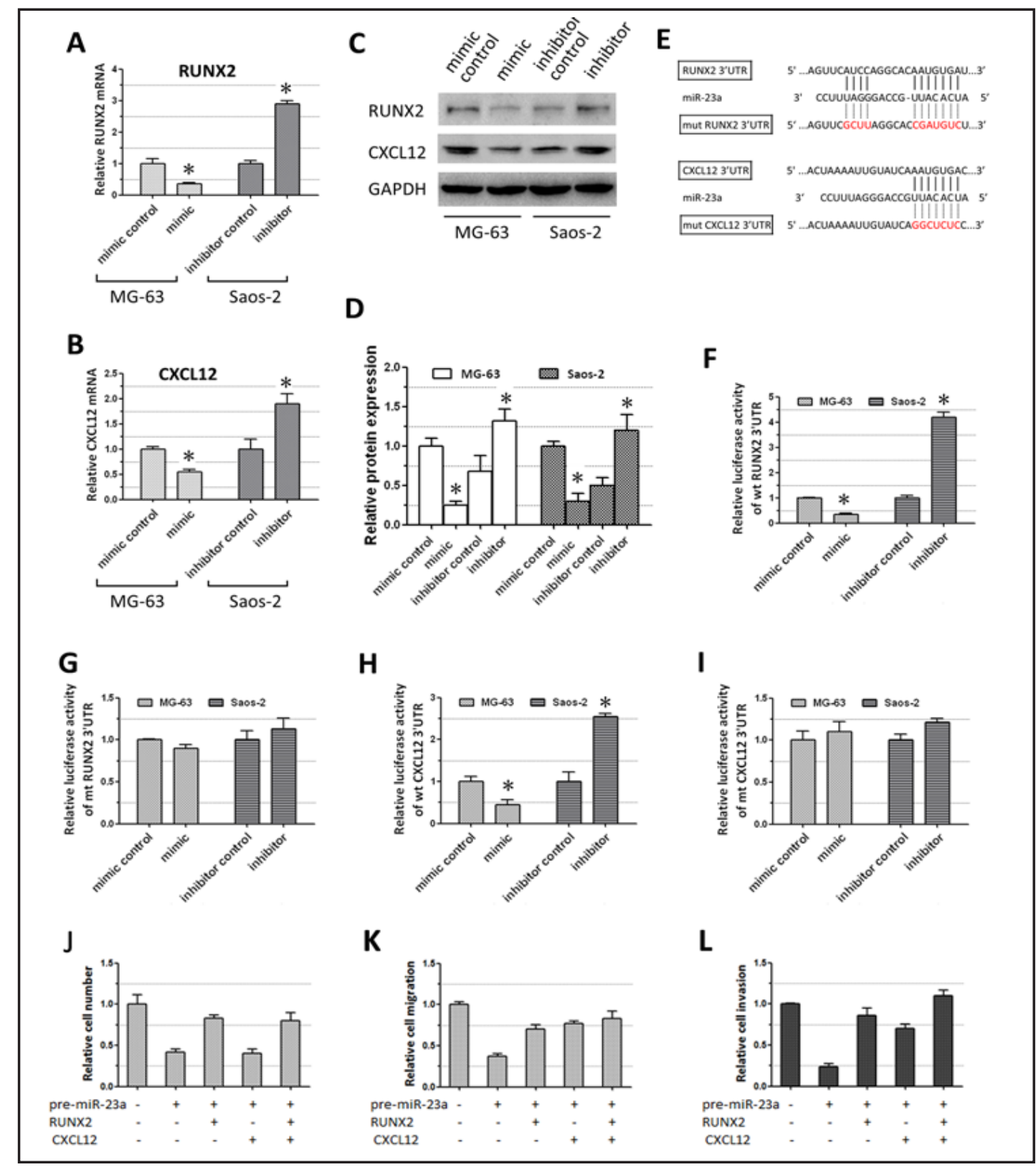

Fig. 4. RUNX2 and CXCL12 are targets of miR-23a. (A) MiR-23a mimic inhibited the expression of RUNX2 mRNA, whereas miR-23a inhibitor increased the expression of RUNX2 mRNA. The indicated cells were transfected with miR-23a mimic, inhibitor or controls, and mRNA was extracted for quantitative real-time PCR analysis after $24 \mathrm{~h}$. (B) MiR-23a mimic inhibited the expression of CXCL12 mRNA, whereas miR-23a inhibitor increased the expression of CXCL12 mRNA. (C) MiR-23a mimic inhibited the expression of RUNX2 and CXCL12 protein, whereas miR-23a inhibitor increased the expression of RUNX2 and CXCL12 protein. The indicated cells were transfected with miR-23a mimic, inhibitor or controls and the cells were collected for Western blot analysis after $48 \mathrm{~h}$. (D) Quantification of protein expression. (E) Schematic representation of the various 3'UTR luciferase reporters used in the following experiments. (F) MiR-23a mimic inhibited the luciferase activity of a construct containing the wild type RUNX2 3'UTR, whereas miR-23a inhibitor increased the luciferase activity of a construct containing the wild type of RUNX2 3'UTR. (G) The luciferase activity of a construct containing the mutant RUNX2 3'UTR was not affected by the miR-23a mimic or inhibitor. $(\mathrm{H})$ Changes in the luciferase activity of the luciferase construct containing a wild type CXCL12 3'UTR caused by miR-23a mimic or inhibitor. (I) The luciferase activity of the luciferase construct containing the mutant CXCL12 3'UTR were not affected. (J) RUNX2 and CXCL12 rescued the retarded cell proliferation 
induced by miR-23a. MG-63 cells were transfected with pre-miR-23a, with or without a RUNX2 or CXCL12 plasmid, cell numbers were determined after $48 \mathrm{~h}$. RUNX2 and CXCL12 rescued the decreased cell migration $(\mathrm{K})$ and invasion (L) induced by miR-23a. *, p < 0.05 vs the control groups.

A

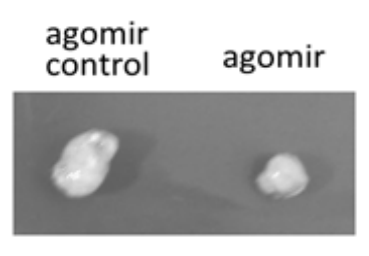

MG-63

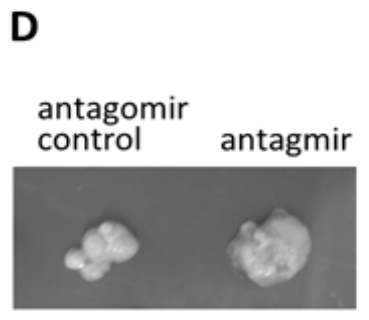

Saos-2
B
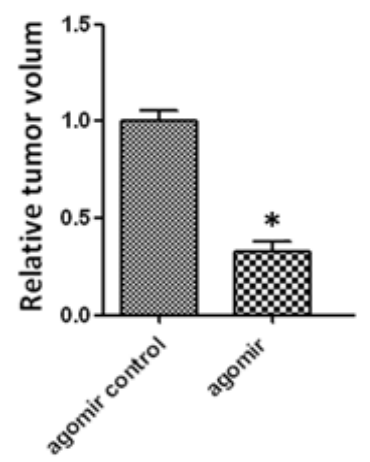

E

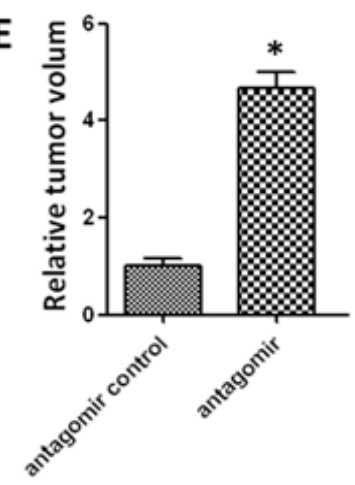

C
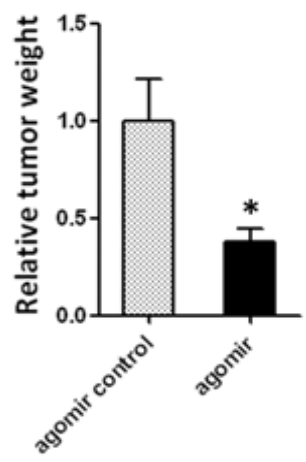

$\mathbf{F}$

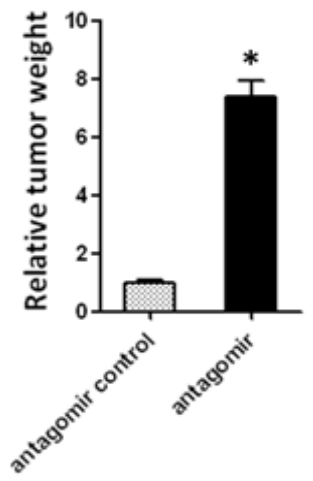

Fig. 5. MiR-23a inhibited OS tumor growth in nude mice. (A) MiR-23a agomir suppressed the growth of MG-63 cells in vivo. MG-63 cells were inoculated into the nude mice, and miR-23a agomir was injected intratumorally after 5 days. Four weeks later the mice were sacrificed to detect the size of the tumor. (D) MiR-23a antagomir stimulated the growth of Saos-2 cells in vivo. (B), (E) The volume of the tumors. (C), (F) The weight of tumors. $*, \mathrm{p}<0.05$ vs the control groups.

2 cells (Fig. 3 F). A quantification of this effect is shown in Fig. $3 \mathrm{G}(\mathrm{p}<0.05)$. Taken together, these results demonstrated that miR-23a inhibited the proliferation, migration, and invasion of OS cells.

\section{RUNX2 and CXCL12 are targets of miR-23a}

MiRNAs regulate the abundance and translation of their target mRNAs. Therefore, the genes that an miRNA regulates are important for the function of an miRNA. We analyzed the potential targets of miR-23a using online software. Several genes were identified as potential targets, and we tested whether these genes were modulated by miR-23a using realtime PCR. Through this experiment, we found that the mRNA levels of RUNX2 and CXCL12 were affected when the expression of miR-23a was modified. As shown in Fig. 4 A, miR-23a mimic decreased the level of RUNX2 mRNA whereas inhibitor of miR-23a increased the level of RUNX2 mRNA $(\mathrm{p}<0.05)$. The CXCL12 mRNA level was similarly changed by the miR23a mimic and inhibitor (Fig. $4 \mathrm{~B}, \mathrm{p}<0.05$ ). The results getting from Western blot analysis demonstrated that the changes in the protein levels of RUNX2 and CXCL12 were consistent with those of the 
corresponding mRNAs (Fig. $4 \mathrm{C}$ and Fig. $4 \mathrm{D}, \mathrm{p}<0.05$ ). To further verify whether RUNX2 and CXCL12 were regulated by miR-23a, we constructed luciferase plasmids containing the 3' UTRs or mutated 3' UTRs of RUNX2 and CXCL12. The complementary regions in the 3' UTRs of RUNX2 and CXCL12 are shown in Fig. 4 E. The luciferase activities of cells treated with either mimic or inhibitor of miR-23a were tested. As shown in Fig. 4 F, transfecting MG-63 cells with miR-23a mimic downregulated the acitivity of luciferase $(p<0.05)$. On the contrary, inhibitor of miR-23a caused a dramatic elevation of luciferase activities $(p<0.05)$. However, neither the mimic nor the inhibitor of miR-23a changed the luciferase activities of constructs containing the mutant RUNX2 3' UTR (Fig. 4 G). The luciferase activity of the 3' UTR of CXCL12 was similarly affected, as shown in Fig. $4 \mathrm{H}$ and Fig. 4 I. Rescue experiments demonstrated that RUNX2 and CXCL12 could rescue the impaired cell proliferation, migration, and invasion induced by miR-23a (Fig. $4 \mathrm{~J}$-L). Taken together, these data suggested that RUNX2 and CXCL12 are targets of miR-23a and that miR-23a regulates the mRNA and protein levels of RUNX2 and CXCL12 to exert its role.

MiR-23a inhibit OS tumor growth in nude mice

Finally, we examined the effect of miR-23a on the tumorigenicity of MG-63 and Saos2 cells in nude mice. The mice were subcutaneously injected with MG-63 cells, a miR23a agomir was injected intratumourally after 5 days, and the primary tumor sizes were monitored after 4 weeks. As shown in Fig. 5 A - Fig. 5 C, treatment with the miR-23a agomir led to a nearly $60 \%$ reduction in tumor volume and tumor weight compared to the controls $(\mathrm{p}<0.05)$. Consistently, after injection of miR-23a antagomir, the average tumor size and weight formed by Saos-2 cells was increased at least 3-fold (Fig. 5 D - Fig. 5 F, p<0.05). These in vivo findings were consistent with the in vitro results observed in the cell proliferation assays, demonstrating that miR-23a robustly blocked tumor growth in OS.

\section{Discussion}

Osteosarcoma is the most common primary bone tumor in children and adolescents. The prognosis for patients with osteosarcoma is poor. Previous data suggestes that $30 \%$ of osteosarcoma patients will not survive for more than 5 years, and less than $50 \%$ will survive for more than 10 years. Studies have reported several genetic alterations in osteosarcoma cells, including chromosomal abnormalities, mutations in tumor suppressor genes or oncogenes, and epigenetic modifications, but the underlying molecular mechanisms of the development of osteosarcoma remain poorly understood. The development of effective treatment strategies or new biomarkers remains an important but unmet clinical need.

MicroRNAs are important gene regulators that play essential roles in regulating the progression of carcinomas including osteosarcoma. Because of their relative ease of detection, altered miRNAs expression profiles may be used to exploit promising targets for diagnosis. Many miRNAs have been found to be upregulated or downregulated in osteosarcoma, such as miR-17-92, miR-145, let-7a, miR-143, miR-181a, and others. However, until now there was no report regarding the dysregulation of miR-23a in osteosarcoma.

In the present study, we found that miR-23a displayed much lower expression levels in osteosarcoma cells and tissues, and that this decrease was due to the DNA methylation of the miR-23a promoter. Functional studies showed that miR-23a functions as a tumor suppressor gene by targeting RUNX2 and CXCL12. Our results revealed for the first time that miR-23a inhibit the growth, migration, and invasion of osteosarcoma cells. The identification of dysregulated miR-23a will likely assist in the development of biomarkers for the progression of osteosarcoma progression.

Recently, numerous lines of evidence have demonstrated that miR-23a is involved in the development of various cancers, including lung cancer, colon cancer, hepatocarcinoma, glioma, breast cancer, colorectal cancer, gastric adenocarcinoma, and haematological malignancies [28-35]. Most of them identified miR-23a as an oncogene due to its 
stimulatory role in cell migration and invasion. For example, miR-23a is regulated by c-MYC and promotes mammary carcinoma cell invasion and hepatic metastasis by targeting Sprouty-2 [32]. MiR-23a also regulates TGF- $\beta$-induced epithelial-mesenchymal transition by targeting E-cadherin in lung cancer cells [28]. However, there were no prior studies reporting a tumor-suppressive function of miR-23a, and we identified miR-23a as tumor suppressor in OS. We speculate that the roles of miR-23a in different types of cancer are tissue-specific and depend upon specific cellular contexts. One of the distinguishing features of osteosarcoma is the destruction of bone, and RUNX2 plays essential roles in the development of osteosarcoma. Our results demonstrated that RUNX2 was one of target genes of miR-23a in OS cells, and the introduction of RUNX2 rescued the impaired proliferation, migration and invasion of OS cells. It is hypothesised that miR-23a plays its tumor suppressor role indirectly, at least in part, by downregulating the expression of RUNX2. Therefore, it was not surprising that miR23a functions as a tumor suppressor in osteosarcoma cells. In other cancer types, such as lung cancer, we did not observed any regulation of RUNX2 by miR-23a. Therefore, in lung cancer, miR-23a may stimulate the progression of cancer and play a different role than it does in OS cells, potentially through mechanisms other than RUNX2.

DNA methylation is one important mechanism of gene regulation. DNA can be modified by the addition of a methyl group to its 5-position of cytosine by DNA methyltransferases (DNMTs). DNA methylation, which is frequently observed on CpG islands, contributes to the transcriptional silencing of genes. The promoter methylation of tumor suppressor genes is commonly observed in carcinoma, and is the predominant mechanism for the loss of tumor suppressor function. In our study, we demonstrated that the promoter region of miR-23a was hypermethylated in osteosarcoma cell lines and tissues, which neatly explains the decreased expression of miR-23a. Therefore, the expression of miR-23a and its promoter methylation represent new biomarker for the specific detection of osteosarcoma. Genomewide DNA methylation analysis will be needed to provide extensive information for exploring miR-23a or other microRNAs as potential diagnostic markers for osteosarcoma. Correlating the methylation status of the miR-23a promoter with the clinocopathologic and molecular profiles from a large OS cohort will also strengthen the evidence in favor of miR-23a as a new biomarker for the specific detection of osteosarcoma.

RUNX2 is a key transcriptional factor that is involved in the differentiation of osteoblasts and chondrocytes. Increasing evidence shows that RUNX2 plays essential roles in certain types of cancer, such as breast cancer and osteosarcoma. These cancers possess some "osteomimic" features and are able to invade into the bone tissues. RUNX2, acting as an oncogene, promotes the proliferation, migration, and invasion of cancer cells. CXCL12, also known as SDF-1, is a potent chemoattractant factor that is important for cancer cell migration. Our results demonstrated that miR-23a targets RUNX2 and CXCL12, and the introduction of RUNX2 and CXCL12 rescued the impaired proliferation, migration and invasion of OS cells, revealing one mechanism that underlies the inhibitory role of miR-23a in osteosarcoma. A single miRNA can regulate the mRNA stability or translation of multiple mRNAs, while one gene can be regulated by multiple miRNAs. Therefore, it is also possible that other miRNAs could also regulate RUNX2 or CXCL12. Therefore, other miRNAs may have roles similar to that of miR-23a in osteosarcoma.

Taken together, we have observed for the first time that the expression of miR-23a is decreased in osteosarcoma and revealed its role as a tumor suppressor gene in these cells. Our data help to elucidate the mechanism of osteosarcoma progression, and demonstrated the possibility of using miR-23a as a useful diagnostic marker for osteosarcoma.

\section{Disclosure Statement}

The authors state that they have no conflicts of interest. 


\section{Acknowledgements}

We thank Dr. Jia LIN for the revision of the manuscript.

\section{References}

Forscher C, Mita M, Figlin R: Targeted therapy for sarcomas. Biologics 2014;8:91-105.

Botter SM, Neri D, Fuchs B: Recent advances in osteosarcoma. Curr Opin Pharmacol 2014; 16C:15-23. Gill J, Ahluwalia MK, Geller D, Gorlick R: New targets and approaches in osteosarcoma. Pharmacol Ther 2013;137:89-99.

4 Gorlick R, Janeway K, Lessnick S, Randall RL, Marina N; COG Bone Tumor Committee: Children's Oncology Group's 2013 blueprint for research: bone tumors. Pediatr Blood Cancer 2013;60:1009-1015.

-5 Bölling T, Hardes J, Dirksen U: Management of bone tumours in paediatric oncology. Clin Oncol (R Coll Radiol) 2013;25:19-26.

6 Szuhai K, Cleton-Jansen AM, Hogendoorn PC, Bovée JV: Molecular pathology and its diagnostic use in bone tumors. Cancer Genet 2012;205:193-204.

7 McManus MT, Sharp PA: Gene silencing in mammals by small interfering RNAs. Nat Rev Genet 2002;3:737747.

-8 Eini R, Dorssers LC, Looijenga LH: Role of stem cell proteins and microRNAs in embryogenesis and germ cell cancer. Int J Dev Biol 2013;57:319-332.

-9 Kuppusamy KT, Sperber H, Ruohola-Baker H: MicroRNA regulation and role in stem cell maintenance, cardiac differentiation and hypertrophy. Curr Mol Med 2013;13:757-764.

10 Choi E, Choi E, Hwang KC: MicroRNAs as novel regulators of stem cell fate: World J Stem Cells 2013;5:172187.

11 Bakhshandeh B, Soleimani M, Paylakhi SH, Ghaemi N: A microRNA signature associated with chondrogenic lineage commitment. J Genet 2012;91:171-182.

12 Shah NR, Chen H: MicroRNAs in pathogenesis of breast cancer: Implications in diagnosis and treatment. World J Clin Oncol 2014;5:48-60.

13 Drusco A, Nuovo GJ, Zanesi N, Di Leva G, Pichiorri F, Volinia S, Fernandez C, Antenucci A, Costinean S, Bottoni A, Rosito IA, Liu CG, Burch A, Acunzo M, Pekarsky Y, Alder H, Ciardi A, Croce CM: MicroRNA Profiles Discriminate among Colon Cancer Metastasis. PLoS One 2014;9:e96670.

14 Ishiguro H, Kimura M, Takeyama H: Role of microRNAs in gastric cancer. World J Gastroenterol 2014;20:5694-5699.

15 D‘Anzeo M, Faloppi L, Scartozzi M, Giampieri R, Bianconi M, Del Prete M, Silvestris N, Cascinu S: The role of Micro-RNAs in Hepatocellular Carcinoma: From Molecular Biology to Treatment. Molecules 2014;19:63936406.

16 Kang SM, Lee HJ: MicroRNAs in human lung cancer: Exp Biol Med (Maywood) 2014; pii: 1535370214533887.

17 Nugent M: MicroRNA function and dysregulation in bone tumors: the evidence to date. Cancer Manag Res 2014;6:15-25.

18 Miao J, Wu S, Peng Z, Tania M, Zhang C: MicroRNAs in osteosarcoma: diagnostic and therapeutic aspects. Tumour Biol 2013;34:2093-2098.

19 Zhou G, Shi X, Zhang J, Wu S, Zhao J: MicroRNAs in osteosarcoma: from biological players to clinical contributors, a review. J Int Med Res 2013;41:1-12.

20 Liang W, Gao B, Fu P, Xu S, Qian Y, Fu Q: The miRNAs in the pathgenesis of osteosarcoma. Front Biosci (Landmark Ed) 2013;18:788-794.

21 Wang J, Zhang KY, Liu SM, Sen S: Tumor-associated circulating microRNAs as biomarkers of cancer. Molecules 2014;19:1912-1938.

22 Palanichamy JK, Rao DS: miRNA dysregulation in cancer: towards a mechanistic understanding. Front Genet 2014; :54. eCollection 2014.

23 Bouyssou JM, Manier S, Huynh D, Issa S, Roccaro AM, Ghobrial IM: Regulation of microRNAs in cancer metastasis. Biochim Biophys Acta 2014;1845:255-265.

24 You JS, Jones PA: Cancer genetics and epigenetics: two sides of the same coin? Cancer Cell 2012;22:9-20. 
25 Akhavan-Niaki H, Samadani AA: DNA methylation and cancer development: molecular mechanism. Cell Biochem Biophys 2013;67:501-513.

-26 Jones PA, Liang G: Rethinking how DNA methylation patterns are maintained. Nat Rev Genet 2009;10:805811.

27 Subramaniam D, Thombre R, Dhar A, Anant S: DNA Methyltransferases: A Novel Target for Prevention and Therapy. Front Oncol 2014;4:80. eCollection 2014.

28 Cao M, Seike M, Soeno C, Mizutani H, Kitamura K, Minegishi Y, Noro R, Yoshimura A, Cai L, Gemma A: MiR23a regulates TGF- $\beta$-induced epithelial-mesenchymal transition by targeting E-cadherin in lung cancer cells. Int J Oncol 2012;41:869-875.

-29 Ogata-Kawata H, Izumiya M, Kurioka D, Honma Y, Yamada Y, Furuta K, Gunji T, Ohta H, Okamoto H, Sonoda H, Watanabe M, Nakagama H, Yokota J, Kohno T, Tsuchiya N: Circulating exosomal microRNAs as biomarkers of colon cancer. PLoS One 2014;9:e92921.

30 Bao L, Zhao J, Dai X, Wang Y, Ma R, Su Y, Cui H, Niu J, Bai S, Xiao Z, Yuan H, Yang Z, Li C, Cheng R, Ren X: Correlation between miR-23a and onset of hepatocellular carcinoma. Clin Res Hepatol Gastroenterol 2014; 38:318-330.

31 Hu X, Chen D, Cui Y, Li Z, Huang J: Targeting microRNA-23a to inhibit glioma cell invasion via HOXD10. Sci Rep 2013;3:3423.

-32 Li X, Liu X, Xu W, Zhou P, Gao P, Jiang S, Lobie PE, Zhu T: c-MYC-regulated miR-23a/24-2/27a cluster promotes mammary carcinoma cell invasion and hepatic metastasis by targeting Sprouty2. J Biol Chem 2013;288:18121-18133.

-33 Wang Z, Wei W, Sarkar FH: miR-23a, a critical regulator of „migR“ation and metastasis in colorectal cancer. Cancer Discov 2012;2:489-491.

-34 Zhu LH, Liu T, Tang H, Tian RQ, Su C, Liu M, Li X: MicroRNA-23a promotes the growth of gastric adenocarcinoma cell line MGC803 and downregulates interleukin-6 receptor. FEBS J 2010;277:3726-3734.

35 Wang WL1, Yang C, Han XL, Wang R, Huang Y, Zi YM, Li JD: MicroRNA-23a expression in paraffin-embedded specimen correlates with overall survival of diffuse large B-cell lymphoma. Med Oncol 2014;31:919. 